\title{
Comparison of phenotype, chondrogenic and osteogenic potential of rheumatoid mesenchymal stem cells derived from articular and subcutaneous adipose tissue - the role of adipocytokines
}

\author{
URSZULA SKALSKA, EWA KONTNY
}

Department of Pathophysiology and Immunology, Institute of Rheumatology, Warsaw, Poland

\begin{abstract}
A study was carried out to compare the phenotype, chondrogenic and osteogenic potential of adipose mesenchymal stem cells (ASCs) derived from articular adipose tissue (AAT) and subcutaneous adipose tissue (SCAT) of rheumatoid arthritis (RA) patients. We also aimed to evaluate the role of leptin $(L E P)$, low molecular weight adiponectin isoform $(L M W)$, high molecular weight adiponectin isoform $(H M W)$ and tumor necrosis factor (TNF) in ASCs differentiation. AAT and ScAT were obtained from RA patients undergoing total knee joint replacement surgery. ASCs were isolated and expanded in vitro. Cells phenotype was assesed by flow cytometry; leptin and adiponectin receptors $m R N A$ expression by RT-PCR. Differentiation was performed in chondrogenic and osteogenic medium with or without LEP, $L M W, H M W$ and TNF. After differentiation, expression of mRNA for Sox9, aggrecan (Agr), collagen $2 a$ (Col2a) for chondrogenesis, and BMP-2, Runx-2 and osteopontin (OPN) for osteogenesis was evaluated by RT-PCR. Dickkopf-1 (DKK-1) and osteoprotegerin (OPG) proteins concentrations were measured in supernatants from osteogenic cultures using ELISA. AAT and ScAT-ASCs have the same $C D 105+C D 90+C D 73+C D 45-C D 34+/-C D 19-C D 14-$ phenotype. AAT-ASCs seem to be more susceptible to chondrogenesis and osteogenesis in vitro. Adipocytokines do not exert strong effect on ASCs differentiation, however HMW slightly increased expression of chondrogenesis markers mRNA in AATASCs. TNF diminished expression of all chondrogenesis markers and OPN, but enhanced expression of BMP-2 and Runx-2 mRNA.
\end{abstract}

Key words: adipose-derived mesenchymal stem cells, subcutaneous adipose tissue, articular adipose tissue, rheumatoid arthritis, chondrogenesis, osteogenesis, adiponectin, leptin, TNF.

(Centr Eur J Immunol 2013; 38 (1): 62-69)

\section{Introduction}

Adipose-derived mesenchymal stem cells (ASCs) have the ability to differentiate into many cell types, including chondrocytes and osteoblasts [1] which make them especially promising in rheumatoid arthritis (RA) treatment. Rheumatoid arthritis is a chronic autoimmune disease characterized by articular cartilage and bone destruction. Currently, adipose tissue and adipocytokines have gained much attention of rheumatologists. Serum concentration of classical adipocytokines like leptin and adiponectin are altered in RA patients comparing to healthy individuals, but their role in this disease is still unclear [2].

Moreover, adiponectin which exists in several isoforms, seems to exert diverse activity depending on its structure $[3,4]$. Tumor necrosis factor plays a pivotal role in RA pathogenesis. It is secreted by immune cells and by adipose tissue as well [2]. All these agents may influence ASCs dif-

Correspondence: Urszula Skalska, Institute of Rheumatology, Department of Pathophysiology and Immunology, Spartańska 1, 02-637 Warsaw, Poland, phone/fax: +48 2284425 40, phone: +48 2264650 07, e-mail: urszula.skalska@ir.ids.pl 
ferentiation potential and therefore influence the disease course. ASCs localize in different fat depots; here we studied ASCs derived from articular adipose tissue (AAT) consisting of knee infrapatellar fat pad (IPFP), and ASCs from periarticular subcutaneous adipose tissue (ScAT). Located in the rheumatoid joint IPFP may be more influenced by the course of inflammatory process than ScAT. Although we have previously found some differences in spontaneous secretory activity of rheumatoid AAT and ScAT, both tissues were highly reactive to proinflammatory stimuli [5].

The objective of this study was to compare the phenotype, chondrogenic and osteogenic potential of rheumatoid ASCs from AAT and ScAT as well as to evaluate the influence of leptin, low and high adiponectin isoforms and TNF on differentiation capacity of ASCs.

\section{Material and methods Patients}

The group of 12 woman with active RA were selected from Rheumoortopaedic Clinic of Institute of Rheumatology in Warsaw, Poland. All patients gave their written informed consent according to the declaration of Helsinki and the study was approved by the Institute of Rheumatology Ethics Committee.

\section{Cell isolation and culture}

Tissue specimens of AAT and periarticular ScAT were obtained from knee joint of RA patients at the time of total knee joint replacement surgery. AAT was taken from IPFP and ScAT from the site of the suture. In order to isolate ASCs, adipose tissue was cut and digested in $0.25 \%$ trypsin (Sigma-Aldrich, St Louis, MO, USA) solution (20 min, $37^{\circ} \mathrm{C}$, agitation). After that, tissue was filtered, washed in PBS (Biomed, Lublin, Poland) and centrifuged 3 min at $1200 \mathrm{~g}$. The lower stromal vascular fraction (SVF) was harvested and other two centrifugation in PBS were performed. If necessary, the red blood cells were lysed. Cells were seeded onto $25 \mathrm{~cm}^{3}$ culture flasks in DMEM/F12/10\%FCS/penicillin/streptomycin/plasmocin medium (GIBCO, Grand Island, NY, USA; Biochrom, Berlin, Germany; Polfa Tarchomin S.A., Warsaw, Poland; InvivoGen, San Diego, CA, USA; respectively) and cultured in $37^{\circ} \mathrm{C}$. Every 2 or 3 days medium was replaced with a fresh one. All the experiments were done with the $4^{\text {th }}$ passage ASCs.

\section{Flow cytometric analysis}

ASCs were collected from cultures using non-enzymatic cell dissociation solution (Sigma-Aldrich) and washed with FACS buffer (phosphate-buffered saline, $0.1 \% \mathrm{NaN}_{3}$, $1 \%$ FCS). $5 \times 10^{4}$ of cells were resuspended in $50 \mu 1$ of FACS buffer and stained for $30 \mathrm{~min}$ on ice with fluorochrome conjugated murine anti-human monoclonal antibodies against following surface markers: CD90-FITC, CD105-PE, CD73-APC (eBioscience), CD34-PE, CD45-PE,
CD19-PE, CD14-APC (Becton Dickinson). Appropriate isotype controls were used. Cells were analysed using FACSCalibur flow cytometer and CellQuest software (Becton Dickinson).

\section{Leptin and adiponectin receptors mRNA detection}

Cells were washed and total cellular RNA was isolated (RNA II Isolation Kit; Macharey-Nagel, Düren, Germany). cDNA synthesis was performed using 200U of M-MLV reverse transcriptase (Invitrogen, Carlsbad, CA, USA). Expression of mRNA for AdipoR1, AdipoR2 and $O b-R$ (common domain) was quantified by polymerase chain reaction (PCR).

The cDNA was amplified with 1.25 U of Taq Polymerase (Invitrogen, Carlsbad, CA, USA) and $0.625 \mu \mathrm{M}$ of primers, denaturation step was performed at $95^{\circ} \mathrm{C}$ and extension at $72^{\circ} \mathrm{C}$. The primers sequence, annealing temperature, cycles number and product size are given in Table 1. PCR products were size fractionated on agarose gel (Genoplast, Pruszków, Poland) with addition of ethidium bromide (Sigma-Aldrich, St Louis, MO, USA) and photographed under UV transillumination. Quantitive analysis of mRNA expression was assessed relatively to house-keeping gene glyceraldehyde-3-phosphate dehydrogenase (GAPDH) expression using gels analysis software (Kodak Digital Science 1D, New Haven, CT, USA).

\section{Cell differentiation}

For differentiation assays cells were cultured in differentiation media with or without human recombinant: LEP $10 \mathrm{ng} / \mathrm{ml}$ (PeproTech, London, UK), LMW 1 g/ml, HMW $1 \mu \mathrm{g} / \mathrm{ml}$ (BioVendor, Brno, Czech Republic) or TNF 10 ng/ml (R\&D Systems, Minneapolis, MN, USA). Chondrogenesis: $2 \times 10^{5}$ cells were cultured in pellets for 21 days in following medium: DMEM with $1 \mathrm{~g} / \mathrm{ml}$ glucose (GIB$\mathrm{CO}$, Grand Island, NY, USA)/sodium puryvate $110 \mathrm{mg} / \mathrm{l}$ (POCH S.A., Gliwice, Poland)/insulin-transferrin-sodium selenite media supplement 1\% (Sigma-Aldrich, St Louis, MO, USA)/ ascorbate-2-phosphate $0.15 \mathrm{mM}$ (SigmaAldrich, St Louis, MO, USA)/TGF- $\beta 110$ ng/ml (PeproTech, London, UK)/dexamethasone $100 \mathrm{nM}$ (SigmaAldrich, St Louis, MO, USA)/penicilin 100 U/ml (Polfa Tarchomin S.A., Warsaw, Poland)/streptomycin $100 \mu \mathrm{g} / \mathrm{ml}$ (Polfa Tarchomin S.A., Warsaw, Poland). Osteogenesis: $2 \times 10^{4}$ cells were cultured in monolayers, in 12-wells culture plates for 14 days in following medium: DMEM supplemented with $4.5 \mathrm{~g} / \mathrm{ml}$ glucose (GIBCO, Grand Island, NY, USA)/fetal calf serum 10\% (Biochrom, Berlin, Germany)/glycerophosphate $10 \mathrm{mM}$ (Sigma-Aldrich, St Louis, $\mathrm{MO}$, USA)/1.25- $(\mathrm{OH})_{2}$ vitamin $\mathrm{D}_{3} 10 \mathrm{nM}$ (Sigma-Aldrich, St Louis, MO, USA)/ascorbate-2-phosphate $0.2 \mathrm{mM}$ (Sigma-Aldrich)/dexamethasone $10 \mathrm{nM}$ (Sigma-Aldrich, St Louis, MO, USA)/penicilin $100 \mathrm{U} / \mathrm{ml}$ (Polfa Tarchomin S.A., Warsaw, Poland)/streptomycin $100 \mu \mathrm{g} / \mathrm{ml}$ (Polfa Tarchomin S.A., Warsaw, Poland). 
Table 1. Primer sequence, annealing temperature, cycles numbers and product size for RT-PCR reactions

\begin{tabular}{|c|c|c|c|c|c|}
\hline Gene & Forward primer sequence & Reverse primer sequence & $\operatorname{Ta}\left({ }^{\circ} \mathbf{C}\right)$ & $\begin{array}{c}\text { Cycles } \\
\text { number }\end{array}$ & $\begin{array}{l}\text { Product } \\
\text { size (bp) }\end{array}$ \\
\hline AdipoR1 & TCTTCCTCATGGCTGTGATG & AGCACTTGGGAAGTTCCTCC & 62 & 30 & 263 \\
\hline AdipoR2 & GGAGCCATTCTCTGCCTTTC & ACCAGATGTCACATTTGCCA & 62 & 30 & 466 \\
\hline$O b-R$ & TGTTGTGAATGTCTTGTGCC & TGCTCCAGTCACTCCAGATTCC & 60 & 33 & 394 \\
\hline$B M P-2$ & CAGAGACCCACCCCCAGCA & CTGTTTGTGTTTGGCTTGAC & 57 & 33 & 672 \\
\hline Runx 2 & GTTTGTTCTCTGACCGCCTC & CCAGTTCTGAAGCACCTGA & 55 & 30 & 318 \\
\hline$O P N$ & CTAGGCATCACCTGTGCCATACC & CAGTGACCAGTTCATCAGATTCATC & 55 & 30 & 373 \\
\hline $\operatorname{Sox} 9$ & TCCTCAGGCTTTGCGATTT & TGCTCGGGCACTTATTGG & 52 & 30 & 304 \\
\hline$A g r$ & ATGCCCAAGACTACCAGTGG & TCCTGGAAGCTCTTCTCAGT & 55 & 30 & 501 \\
\hline Col2a & TTCAGCTATGGAGATGACAAT & AGAGTCCTAGAGTGACTGAG & 58 & 30 & 432 \\
\hline$G A P D H$ & TGGTATCGTGGAAGGACTCA & ATGCCAGTGAGCTTCCCGTT & 63 & 23 & 189 \\
\hline
\end{tabular}

Ta-annealing temperature, $b p$ - base pair

\section{Osteogenesis and chondrogenesis markers mRNA detection}

After differentiation, expression of mRNA for $\operatorname{Sox} 9$, Agr, Col2a (chondrogenic cultures) and Runx-2, BMP-2 and $O P N$ (osteogenic cultures), was quantified by RT-PCR. The procedure and cells treatment was the same as in section "Leptin and adiponectin receptors detection". The primer sequence, annealing temperature, cycle number and product size are given in Table 1.

\section{Enzyme linked immunosorbent assay (ELISA)}

The concentration of DKK-1 and OPG was measured in supernatants ( $\mathrm{SNs}$ ) from osteogenic cultures using ELISA. SNs were collected after the end of osteoblastic differentiation (on day $14^{\text {th }}$ ), centrifuged and frozen at $-70^{\circ} \mathrm{C}$. All measurements were done in duplicates. DKK-1 measurement was performed using appropriate ELISA kit (DuoSet, R\&D Systems, Minneapolis, MN, USA). OPG measurement was performed using own procedure. 96-wells plates were coated with capture antibody (monoclonal mouse anti-human OPG IgG2A) and incubated overnight at $4{ }^{\circ} \mathrm{C}$. After washing, blocking solution (PBS with $1 \%$ bovine serum albumin) was added for $1 \mathrm{~h}$ at room temperature (RT). Standards and samples were incubated $1 \mathrm{~h}$ at RT, with gentle agitation. Antigen detection was performed using polyclonal gout biotinylated anti-human OPG IgG $(1 \mathrm{~h}, \mathrm{RT})$, than samples were incubated $(20 \mathrm{~min}, \mathrm{RT})$ with streptavidin-horse-radish peroxidase conjugate (all antibodies and human recombinant protein purchased from R\&D Systems, Minneapolis, MN, USA).

Enzymatic reaction was performed using $o$-phenylenediamine dihydrochloride (Sigma-Aldrich, St Louis, MO, USA) as a substrate and stopped with $1 \mathrm{M} \mathrm{H}_{2} \mathrm{SO}_{4}$. Concentration of OPG was measured colorimetrically at $492 \mathrm{~nm}$ (measurement filter) and $620 \mathrm{~nm}$ (reference filter).

\section{Statistical analysis}

All statistical analysis were made using STATISTICA 6.0 software (StatSoft Inc., Tulsa, OK, USA). Data are expressed as means and standard error (SE) unless otherwise noted. The normality of data distribution was assessed by Shapiro-Wilk test. All data was not normally distributed. The Mann-Whitney U test was used for comparison between AAT and ScAT groups. The differences between gene expression in control, differentiated and adipocytokines treated samples within the limits of one group (AAT or ScAT) were evaluated using Wilcoxon signed-rank test. Differences were considered significant for $p<0.05$.

\section{Results \\ Patients}

Demographic and clinical characteristics of the study patients are presented in Table 2 .

None of the study patients were treated with biological therapy.

\section{Phenotypic ASCs analysis}

In order to evaluate the phenotype of ASCs derived from rheumatoid AAT and ScAT, cells were analyzed by flow cytometry. Cells from both tissues were almost completely positive for CD105, CD90 and CD73 expression. Less than $1 \%$ of analyzed cells expressed CD 45, CD19 and CD14, while CD34 was expressed by about $10 \%$ of analyzed cells (Fig. 1A, B). Thus, both AAT-ASCs and ScATASCs derived cells were highly enriched in mesenchymal stem cells.

\section{Adiponectin and leptin receptors mRNA expression}

AdipoR1 and AdipoR2 are both functional and ubiquitousely expressed adiponectin receptors. However Adi- 
poR1 is expressed primarily in skeletal muscle and AdipoR2 in the liver [6]. Ob-R exists in six isoforms derived from alternative splicing from a single gene. All isoforms share common extracellular domain and differ in the length of cytoplasmic domain [7, 8]. Expression of mRNA for AdipoR1, AdipoR2 and common domain of Ob-R were detected in ASCs from both AAT and ScAT. Control reactions without reverse transriptase showed no DNA amplification (Fig. 2).

\section{Chondrogenesis and osteogenesis markers mRNA expression}

The relative differences in chondrogenesis and osteogenesis markers mRNA expression between control and stimulated ASCs as well as between cells from AAT and ScAT were evaluated by RT-PCR method. Expression of mRNA for all studied markers was slightly higher in AATASCs than in ScAT-ASCs cultured in control and differen-
Table 2. Demographic and clinical characteristics of the study patients

\begin{tabular}{lc}
\hline Characteristics & RA patients, $\boldsymbol{n}=\mathbf{1 2}$ \\
\hline Age, mean \pm SD (range), years & $56.1 \pm 7.2(42-67)$ \\
\hline Duration of RA \pm SD, mean (range), years & $18.1 \pm 7.0(7-30)$ \\
\hline DAS28 (range) & $4.34 \pm 0.73(2.95-5.67)$ \\
\hline High severity RA (DAS28 > 5.1), n (\%) & $2(16.7)$ \\
\hline $\begin{array}{lc}\text { Moderate severity RA (DAS28 3.2 - 5.1), } \\
n(\%)\end{array}$ & $9(75)$ \\
\hline Low severity RA (DAS28 < 3.2), $n(\%)$ & $1(8.3)$ \\
\hline CRP, mean \pm SD (range), mg/litre & $25.9 \pm 23.1(6-84)$ \\
\hline ESR 1 h, mean \pm SD (range) & $31.1 \pm 17.3(6-58)$ \\
\hline
\end{tabular}

$C R P$-C-reactive protein; ESR - erythrocyte sedimentation rate

B

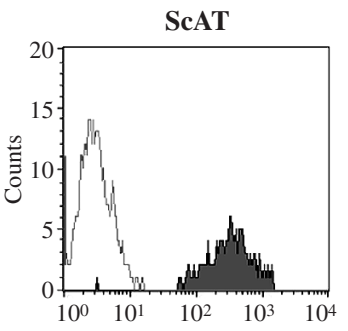

A
AAT
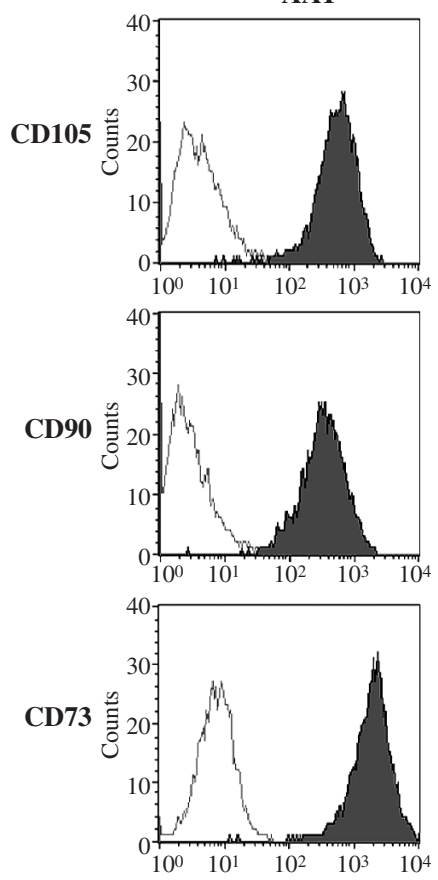
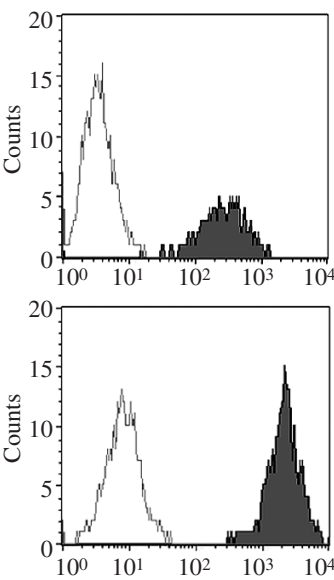

\begin{tabular}{lrr}
\hline & \multicolumn{1}{c}{ AAT } & \multicolumn{1}{c}{ ScAT } \\
\hline CD105 & $98.56(1.06)$ & $97.90(2.23)$ \\
CD90 & $98.58(0.85)$ & $98.08(1.91)$ \\
CD73 & $98.51(0.46)$ & $98.17(1.41)$ \\
CD45 & $0.05(0.05)$ & $0.06(0.08)$ \\
CD34 & $10.47(4.74)$ & $6.36(9.18)$ \\
CD19 & $0.09(0.18)$ & $0.03(0.05)$ \\
CD14 & $0.05(0.07)$ & $0.03(0.05)$ \\
\hline
\end{tabular}
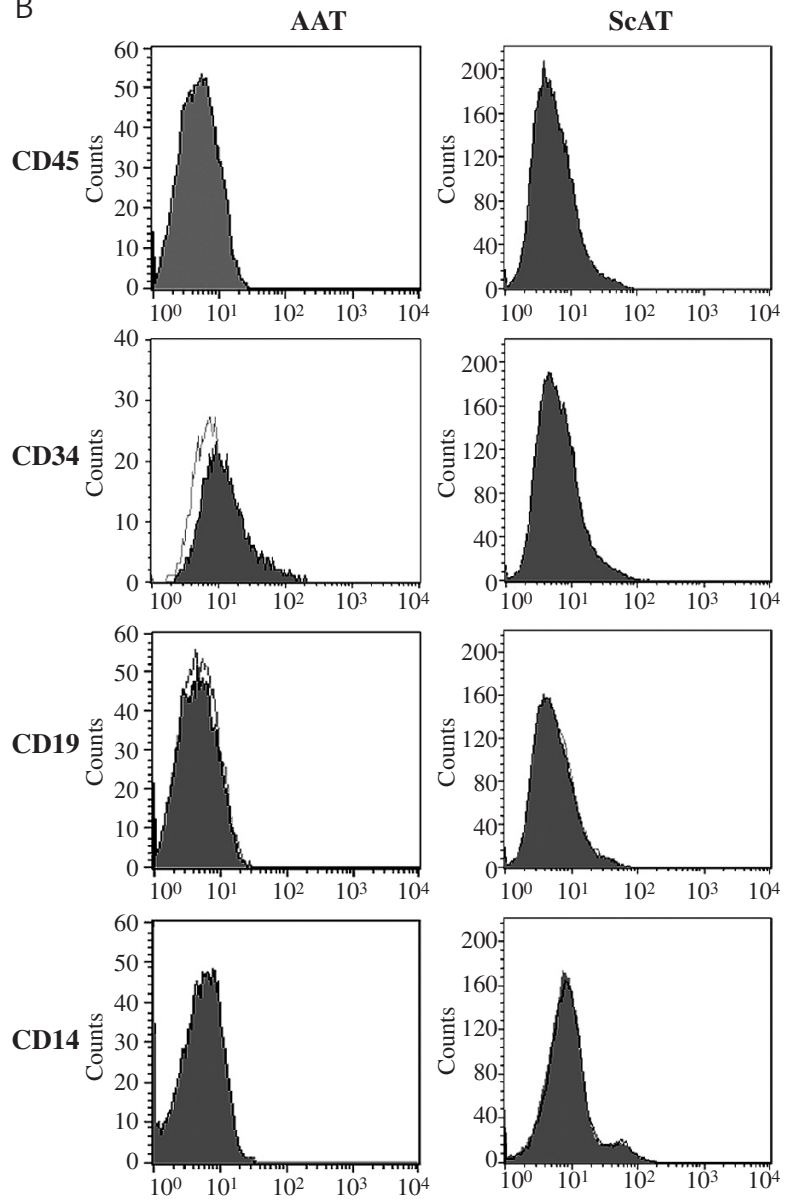

Fig. 1A, B. Cytometric characteristic of rheumatoid ASCs from AAT and ScAT. A) Grey closed histograms represent expression of tested molecules, grey-line histograms represent isotypematched controls. B) Percentage of cells positive for each marker; values are the mean (SD) of 4 or 5 independent experiments 
tiation media, but these differences did not reached statistical significance except Runx2 (Figs. 3, 4). In AAT-ASCs cultured in chondrogenic medium, we observed up-regulation of all chondrogenesis markers, whereas in ScAT-ASCs only Col $2 a$ mRNA expression was upregulated.

HMW adiponectin treatment further increased the expression of Sox 9 and Col2a mRNA in AAT-ASCs, but not in ScAT-ASCs. In ASCs derived from both tissues TNF caused down-regulation of all markers mRNA comparing to the chondrogenic mediumtreated cells (Fig. 3).

In AAT-ASCs expression of mRNA for Runx 2 and $O P N$ was significantly, while $B M P$-2 slightly up-regulated after osteogenic medium treatment. In these cells TNF further raised Runx 2 and BMP-2 mRNA levels while inhibited $O P N$ expression. ScAT-ASCs failed to express BMP-2 and upon treatment with osteogenic medium only $O P N$ mRNA upregulation was obsereved in these cells. Similar to AATASCs, also in ScAT-ASCs TNF counteracted the increase of $O P N$ but elevated Runx 2 mRNA expression. Among adipokines, only LMW adiponectin had any effects: it upregulated $B M P-2$ expression in AAT-ASCs and downregulated $O P N$ in ScAT-ASCs (Fig. 4).

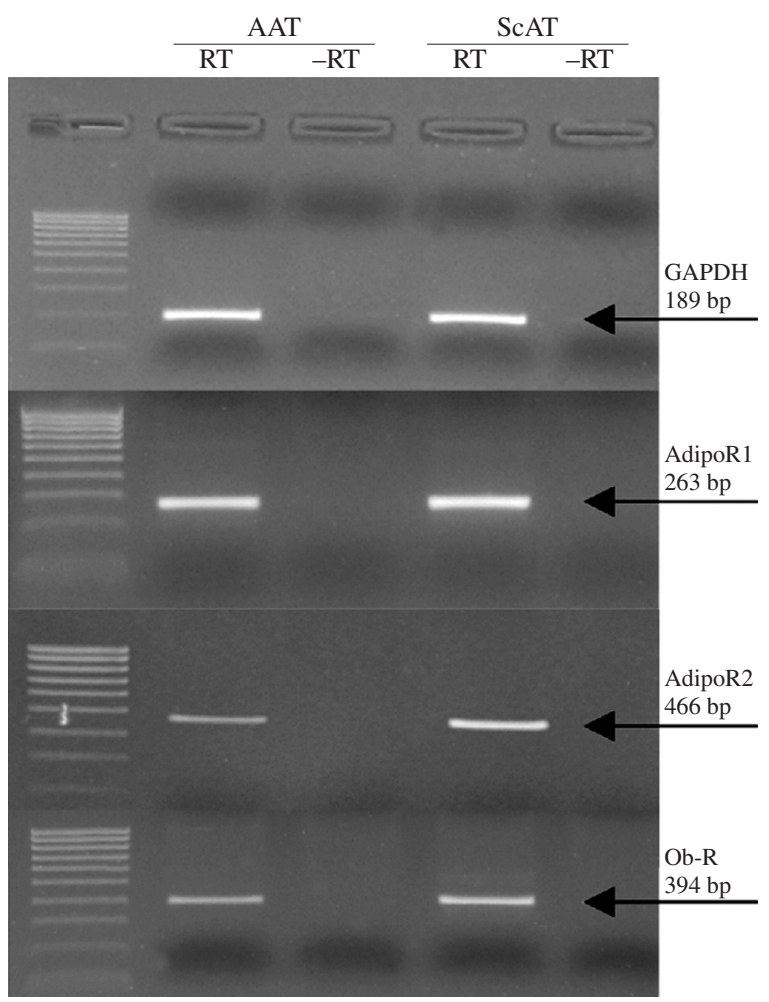

Fig. 2. Expression of mRNA for AdipoR1, AdipoR2 and ObR common domain in rheumatoid ASCs form AAT and ScAT. Representative RT-PCR results demonstrating products obtained in reaction with reverse transcriptase (RT) and without reverse transcriptase (-RT). Expression of mRNA for house-keeping gene GAPDH and PCR products length in base pairs (bp) are also shown
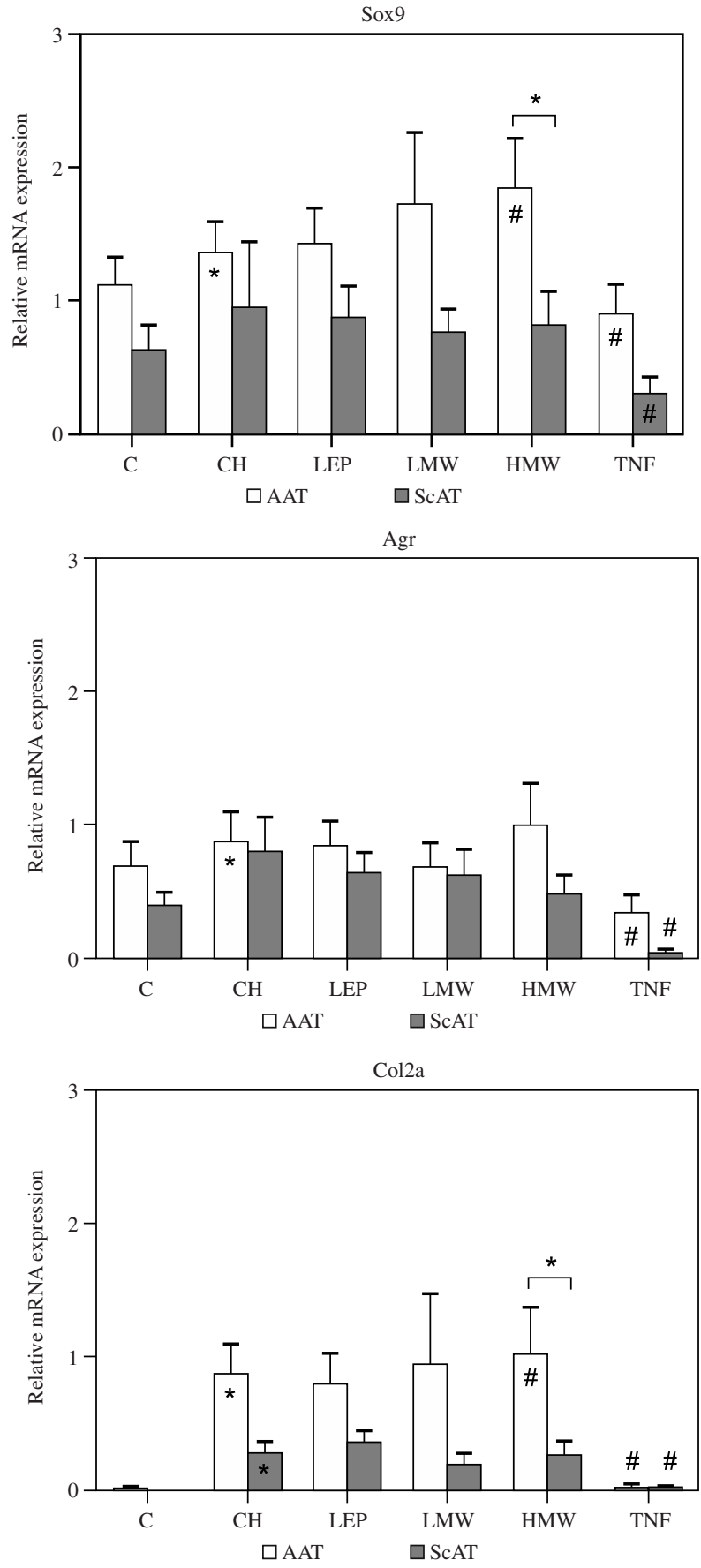

Fig. 3. Expression of Sox 9 , Agr and Col2a mRNA in rheumatoid ASCs from AAT and ScAT cultured in control (C), chondrogenic $(\mathrm{CH})$, chondrogenic $+10 \mathrm{ng} / \mathrm{ml}$ leptin (LEP), chondrogenic $+1 \mu \mathrm{g} / \mathrm{ml} \mathrm{LMW}$ adiponectin (LMW), chondrogenic $+1 \mu \mathrm{g} / \mathrm{ml} \mathrm{HMW} \mathrm{(HMW)} \mathrm{and} \mathrm{chondrogenic}+10 \mathrm{ng} / \mathrm{ml} \mathrm{TNF}$ (TNF) supplemented media. Values are the mean and SE of 11 (AAT) and 12 (ScAT) independent experiments. Statistical analysis was performed as described in Materials and Methods. Asterisk (*) inside the bars indicates statistical significance vs. control; hash (\#) indicates statistical significance vs. chondrogenic medium; asterisk (*) above the bars indicates statistical significance between AAT and ScAT 

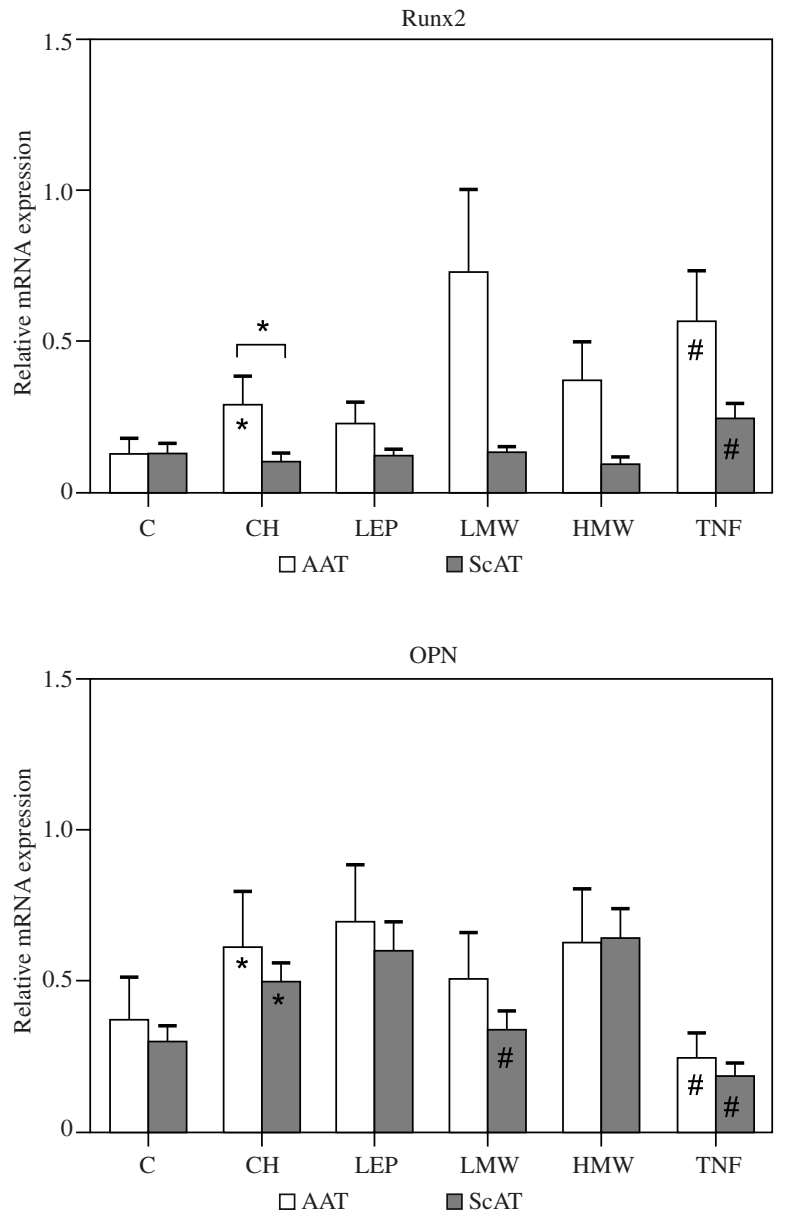

\section{DKK-1 and OPG secretion}

Spontaneous secretion of DKK-1 was higher in ScATASCs than in AAT-ASCs and remained stable after stimulation. In contrary, AAT-ASCs released more DKK-1 after osteogenic medium-treatment and this was potentiated by TNF. ASCs from both tissues released spontaneously comparable amounts of OPG. In AAT-ASCs neither osteogenic medium nor adipokines modified OPG secretion but the release of this cytokine was significantly raised by TNF. Osteogenic medium-treated ScAT-ASCs released considerably less OPG than control cells, which was deepened by LMW adiponectin (Fig. 5).

\section{Discussion}

In presented study we aimed to compare ASCs derived from two different fat depots - AAT and ScAT - regarding their phenotype, chondrogenic and osteogenic potential. The second objective was to evaluate the effects of classical adipokines and TNF on differentiation capacity of ASCs.

We failed to observe differences in the phenotype of ASCs derived from AAT and ScAT. In both tissues more than $97 \%$ of ASCs expressed CD90, CD105 and CD73.

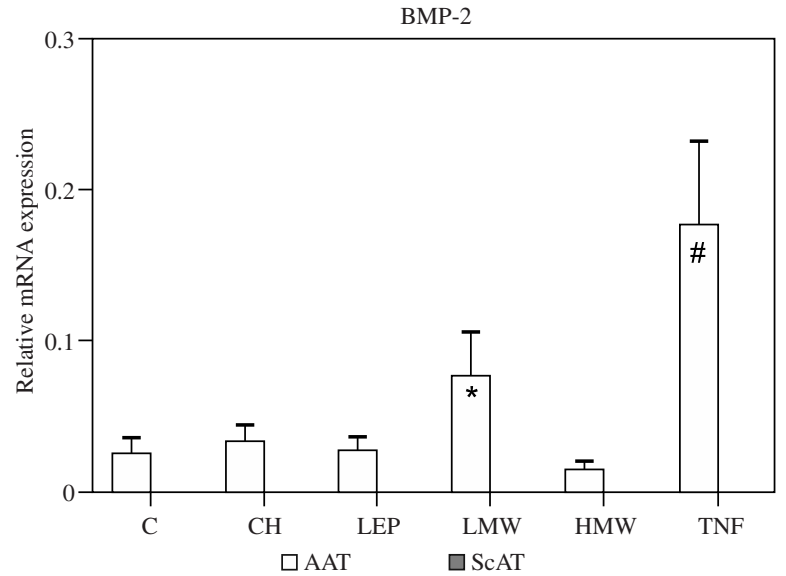

Fig. 4. Expression of $B M P-2, R u n x 2$ and $O P N$ mRNA in rheumatoid ASCs from AAT and ScAT cultured in control (C), osteogenic (OS), osteogenic + $10 \mathrm{ng} / \mathrm{ml}$ leptin (LEP), osteogenic $+1 \mu \mathrm{g} / \mathrm{ml}$ LMW adiponectin (LMW), osteogenic $+\mu \mathrm{g} / \mathrm{ml}$ HMW (HMW) and osteogenic + $10 \mathrm{ng} / \mathrm{ml}$ TNF (TNF) supplemented media. Values are the mean and SE of 11 (AAT) and 12 (ScAT) independent experiments. Statistical analysis was performed as described in Materials and methods. Asterisk (*) inside the bars indicates statistical significance vs. control; hash (\#) indicates statistical significance vs. osteogenic medium; asterisk (*) above the bars indicates statistical significance between AAT and ScAT

CD45, CD19 and CD14 were present at less than $1 \%$ of analyzed cells (Fig. 1) which is in accordance with criteria for defining multipotent mesenchymal stem cells recommended by the International Society for Cellular Therapy [9]. CD34, a classical haematopoietic and endothelial cells marker, was expressed at about $10 \%$ of analyzed cells. The presence of CD34+ cells among ASCs has been already reported elswhere [10-13].

The regenerative properties of ASCs are well documented and these cells can be easily differentiatied toward many cells types [1]. Few reports comparing osteogenic and chondrogenic capacity of mesenchymal stem cells isolated from different fat depots are available. In healthy individuals, ScAT-ASCs and visceral ASCs have been shown to possess similar multigermline potential [14], however the comparison of ScAT-ASCs with adipose synoviumderived ASCs revealed that the latter had better chondrogenic capacity [15]. Analysis of chondrogenic and osteogenic potential of ASCs derived from abdominal subcutaneous layer and greater omentum [16] as well as derived from various subcutaneous locations [17] did not reveal any differences. In our experiments ScAT-ASCs from RA patients expressed slightly less mRNA encoding 

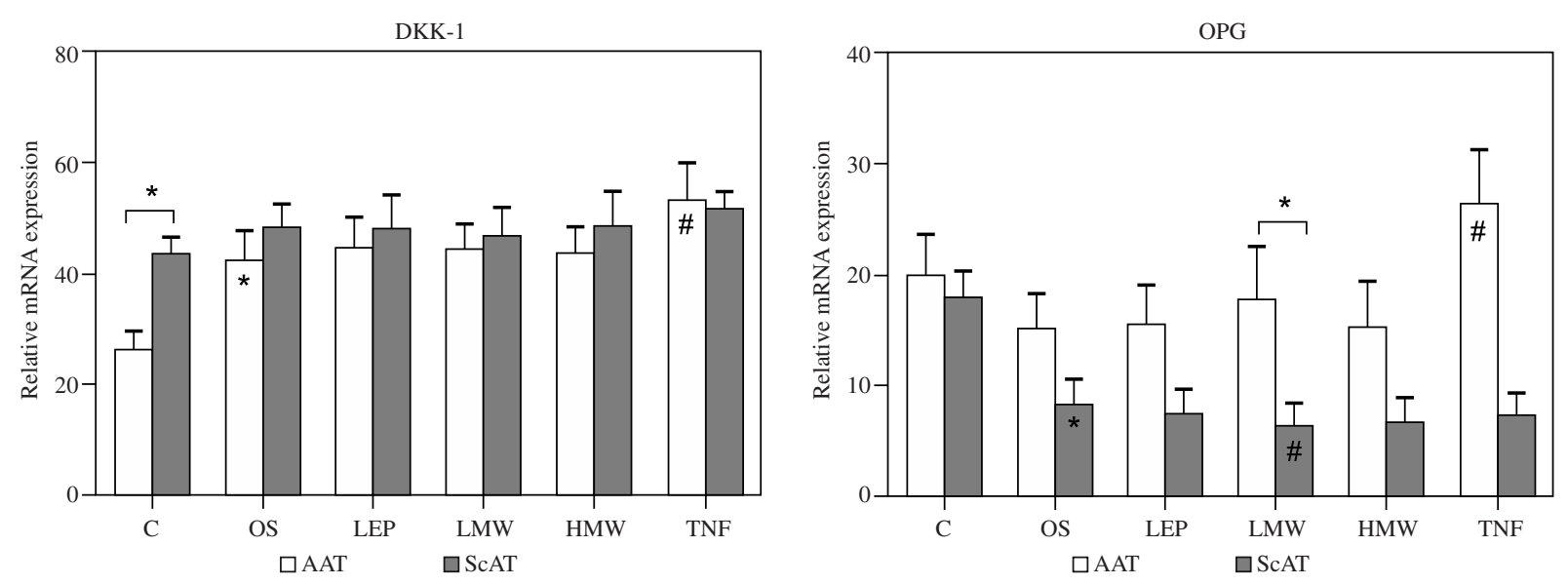

Fig. 5. DKK-1 and OPG secretion by rheumatoid ASCs from AAT and ScAT cultured in control (C), osteogenic (OS), osteogenic $+10 \mathrm{ng} / \mathrm{ml}$ leptin (LEP), osteogenic $+1 \mu \mathrm{g} / \mathrm{ml} \mathrm{LMW} \mathrm{adiponectin} \mathrm{(LMW),} \mathrm{osteogenic}+1 \mu \mathrm{g} / \mathrm{ml} \mathrm{HMW}$ (HMW) and osteogenic $+10 \mathrm{ng} / \mathrm{ml}$ TNF (TNF) supplemented media. Cytokines concentration was measured by ELISA in culture supernatants in duplicates. Values are the mean and SE of 11 (AAT) and 12 (ScAT) independent experiments. Statistical analysis was performed as described in Materials and methods. Asterisk (*) inside the bars indicates statistical significance vs. control; hash (\#) indicates statistical significance vs. osteogenic medium; asterisk (*) above the bars indicates statistical significance between AAT and ScAT

markers of chondrogenic and osteogenic differentiation and were less responsive to chondrogenic and osteogenic media (Figs. 3, 4). Moreover, ScAT-ASCs secreted lower amounts of osteoprotective OPG and more DKK-1, which is well known to inhibit osteoblastogenesis, than AAT-ASCs (Fig. 5). Interestingly, AAT-ASCs and ScAT-ASCs from osteoarthritis (OA) subjects were reported to exhibit similar osteogenic potential [18]. Reassuming, it seems that ASCs derived from rheumatoid articular adipose tissue have stronger differentiation potential despite localization in the site of inflammation.

In order to evaluate the effect of classical adipokines, we have firstly analyzed the expression of mRNA for AdipoR1, AdipoR2 and ObR. mRNA for two isoforms of AdipoR and common domain of ObR were detected in both AAT and ScAT-ASCs (Fig. 2). We did not observed any impact of leptin treatment on the expression of mRNA encoding differentiation markers and proteins secretion by ASCs (Figs. 3-5). HMW adiponectin slightly increased expression of chondrogenic markers in AAT-, but had no effect on ScAT-ASCs (Fig. 3). Stimulation with LMW adiponectin had divers effects on osteogenesis (Figs. 4, 5). This adiponectin isoform decreased the expression of $O P N$ mRNA in ScAT-ASCs and the release of OPG from these cells. By contrast, it had no inhibitory effect on AAT-ASCs and even upregulated $B M P-2$ expression in these cells. Thus, present results suggest that depending on localization, ASCs differ in their reactivity to adiponectin isoforms. Among published data there is no reports concerning adiponectin isoforms effect on these cells differentiation. However, total adiponectin was shown to enhance chon- drocyte differentiation and mesenchymal stem cells osteoblastic differentiation [19, 20].

In our previous publications, we have reported that TNF is able to inhibit chondrogenesis of AAT-ASCs from RA and OA patients [21]. We have also shown that TNF has complex effect on AAT-ASCs osteogenesis: it up-regulates early osteogenesis markers mRNA expression and enhances calcium deposition, but decreases expression of mRNA coding for non-mineral bone components [22]. Here we have demonstrated that ASCs from AAT and ScAT responded similarily to TNF stimulation, which inhibited expression of chondrogenic markers and late osteogenic marker $O P N$, whereas enhanced expression of early osteogenic markers (Run $x 2$ and BMP-2) (Figs. 3, 4). However, OPG and DKK-1 secretion which was up-regulated by TNF in AAT-ASCs, stayed unchanged in ScAT-ASCs (Fig. 5). It is well proved that TNF modifies DKK-1 and OPG secretion but its effect depends on the cell type [23-26]. Our results suggest that in this regard ScAT-ASCs are less susceptible to TNF. Summarizing, we demonstrated that AAT and ScAT-ASCs have similar phenotype, but differ regarding their differentiation capacity. ScAT-ASCs seem to be less competent to undergo osteogenesis and chondrogenesis in vitro than AAT-ASCs. TNF has considerable impact on ASCs differentiation, whereas classical adipokines do not exert strong effect on this process.

We thank dr hab. Barbara Lisowska and prof. dr hab. Pawet Matdyk from Rheumoortopaedic Clinic of Institute of Rheumatology in Warsaw and their patients for providing adipose tissue for these studies. This work was sup- 
ported by a grant from Polish Ministry of Science and High Education No. N N402 369938.

The authors declare no conflict of interest.

\section{References}

1. Zuk P (2010): The adipose-derived stem cell: looking back and looking ahead. Mol Biol Cell 21: 178317-178387.

2. Neumann E, Frommer K, Vasile M, et al. (2011): Adipocytokines as driving forces in rheumatoid arthritis and related inflammatory diseases? Arth Rheum 63: 1159-1169.

3. Neumeier M, Weigert J, Schäffler A, et al. (2006): Different effects of adiponectin isoforms in human mononocytic cells. J Leuk Biol 79: 803-808.

4. Song H, Chan J, Rovin B, et al. (2009): Induction of chemokine expression by adiponectin in vitro is isoform dependent. Trans Res 154: 18-26.

5. Marcinkiewicz J, Kontny E (2012): Taurine and inflammatory diseases. Amino Acids DOI 10.1007/s00726-012-1361-4.

6. Brochu-Gaudreau K, Rehfeldt C, Blouin R, et al. (2010): Adiponectin action from head to toe. Endocrine 37: 11-31.

7. Tartaglia L, Dembski M, Weng X, et al. (1995): Identification and expression cloning of a leptin receptor, OB-R. Cell 83: 1263-1271.

8. Burguera B, Couce M, Long J, et al. (2000): The long form of the leptin receptor $(\mathrm{OBRb})$ is widely expressed in the human brain. Neuroendocrinology 71: 187-195.

9. Dominici M, Le Blanc K, Mueller I (2006): Minimal criteria for defining multipotent mesenchymal stromal cells. The International Society for Cellular Therapy position statement. Cytotherapy 8: 315-317.

10. Lin C, Ning H, Lin G, et al. (2012): Is CD34 truly a negative marker for mesenchymal stromal cells? Cytotherapy 14: 11591163

11. Baer P, Kuci S, Krause M, et al. (2013): Comprehensive phenotypic characterization of human adipose-derived stromal/ stem cells and their subsets by a high throughput technology. Stem Cells Dev 22: 330-339.

12. Lin C, Xin Z, Deng C, et al. (2010): Defining adipose tissuederived stem cells in tissue and in culture. Histol Histopathol 25: 807-815.

13. Zimmerlin L, Donnenberg V, Pfeifer M, et al. (2010): Stromal vascular progenitors in adult human adipose tissue. Cytometry A 77A: 22-30.

14. Baglioni S, Francalanci M, Squecco R, et al. (2009): Characterization of human adult stem-cell populations isolated from visceral and subcutaneous adipose tissue. FASEB J 23: 3404-3505.

15. Mochizuki T, Muneta T, Sakaguchi Y, et al. (2006): Higher chondrogenic potential of fibrous synovium- and adipose synovium-derived cells compared with subcutaneous fat-derived cells: distinguishing properties of mesenchymal stem cells in humans. Arthritis Rheum 2006; 54: 843-853.

16. Requicha JF, Viegas CA, Albuquerque CM, et al. (2012): Effect of anatomical origin and cell passage number on the stemness and osteogenic differentiation potential of canine adiposederived stem cells. Stem Cell Rev 8: 1211-1122.

17. Sachs P, Francis M, Zhao M, et al. (2012): Defining essential stem cell characteristics in adipose-derived stromal cells extracted from distinct anatomical sites. Cell Tissue Res 349: 505-515.

18. Pires de Carvalho P, Hamel KM, Duarte R, et al. (2012): Comparison of infrapatellar and suncutaneous adipose tissue stromal vascular fraction and stromal/stem cells in osteoarthritis subjects. J Tissue Eng Regen Med DOI 10.1002/term.1565.
19. Challa TD, Rais Y, Ornan EM (2010): Effect of adiponectin on ATDC5 proliferation, differentiation and signaling pathways. Mol Cell Endocrinol 323: 282-291.

20. Lee H, Kim S, Kim A, et al. (2009): Adiponectin stimulates osteoblasts differentiation through induction of COX2 in mesenchymal progenitor cells. Stem Cells 27: 2254-2262.

21. Skalska U, Kontny E, Prochorec-Sobieszek M, et al. (2012): Intra-articular adiposederived mesenchymal stem cells from rheumatoid arthritis patients maintain the function of chondrogenic differentiation. Rheumatology 51: 1757-1764.

22. Skalska U, Prochorec-Sobieszek M, Kontny E (2012): Osteoblastic potential of infrapatellar fat pad-derived mesenchymal stem cells from rheumatoid arthritis and osteoarthritis patients. [Manuscript submitted for publication]

23. Heiland G, Zwerina K, Baum W, et al. (2010): Neutralisation of Dkk-1 protects from systemic bone loss during inflammation and reduces sclerostin expression. Ann Rheum Dis 69: 2152-2159.

24. Corallini F, Celeghini C, Rimondi E, et al. (2011): Trail downregulates the release of osteoprotegerin $(\mathrm{OPG})$ by primary stromal cells. J Cell Physiol 226: 2279-2286.

25. Pantoulia E, Boehma M, Kokab S, et al. (2005): Inflammatory cytokines activate $\mathrm{p} 38 \mathrm{MAPK}$ to induce osteoprotegerin synthesis by MG-63 cells. Biochem Biophys Res Commun 329: 224-229.

26. Ann J, Han D, Kim D, et al. (2007): Expression and regulation of osteoprotegerin in adipose tissue. Yonsei Med J 48: 765-772. 